\title{
Oral Administration of Nonionic Water-Soluble Contrast Media to Treat Meconium Obstruction in Premature Infants: A Preliminary Study
}

\author{
Hyun Sook Hong, $\mathrm{MD}, \mathrm{PhD}^{1}$, Sung Shin Kim, $\mathrm{MD}, \mathrm{PhD}^{2}$, and Ga Young Park, $\mathrm{MD}^{2}$ \\ Departments of ${ }^{1}$ Radiology and ${ }^{2}$ Pediatrics, Soonchunhyang University Bucheon Hospital, Soonchunhyang University College of \\ Medicine, Bucheon, Korea
}

\section{ABSTRACT}

Purpose: Meconium obstruction of prematurity (MOP) predisposes premature infants to intestinal perforation and prolonged hospitalization if not diagnosed and treated promptly. A standard contrast enema is less effective to treat infants with distal ileal obstructions because the contrast may not reach the obstructed areas. In an effort to avoid risky surgery, we administered oral contrast media to seven clinically diagnosed patients with MOP whose obstructions were not relieved via conventional sonography-guided contrast enema. We retrospectively evaluated whether oral nonionic water-soluble contrast media relieves MOP.

Methods: Seven of 67 premature infants with MOP were administered oral contrast media from June 2015 to January 2019. Patients were followed-up radiographically for bowel distention and evacuation of contrast media after oral administration. We recorded radiographic improvements, meconium evacuation, time to first feeding after oral contrast media administration, maternal history, and neonatal clinical factors.

Results: We evaluated five male and two female infants. The median gestational ages and body weights at birth were $27^{+5}$ weeks and $890 \mathrm{~g}$, respectively. Radiography in five infants revealed multiple distended intestinal loops without air-fluid interfaces. Two infants had gasless abdomens, in which only stomach gas was visible. Oral contrast media (median, $2.5 \mathrm{~mL}$ ) were administered at a median age of 7 days; five infants $(5 / 7,71.4 \%)$ responded to this treatment. The remaining two infants, who had ileal stenosis and hypoganglionosis, were surgically managed. Five infants (5/7, $71.4 \%$ ) had maternal risk factors, and two (28.6\%) were small for gestational age.

Conclusion: Nonionic oral water-soluble contrast medium can serve as a valuable adjunct treatment in premature infants with meconium obstruction.

Key Words: Meconium; Intestinal obstruction; Contrast media; Infant, premature
Received: 27 August 2020

Revised: 19 October 2020

Accepted: 21 November 2020

Correspondence to: Hyun Sook Hong, $\mathrm{MD}, \mathrm{PhD}$

Department of Radiology, Soonchunhyang University Bucheon Hospital, Soonchunhyang University College of Medicine, 170 Jomaru-ro, Wonmi-gu, Bucheon 14584, Korea

Tel: +82-32-621-5851

Fax: +82-32-621-5874

E-mail:hshong@schmc.ac.kr

Copyright(c)

By Korean Society of Neonatology. All right reserved.

This is an Open-Access article distributed under the terms of the Creative Commons At tribution Non-Commercial License (http:// creativecommons.org/licenses/by-nc/4.0), which permits unrestricted non-commercial use, distribution, and reproduction in any medium, provided the original work is properly cited. 


\section{INTRODUCTION}

Meconium abnormalities in neonates may cause intestinal obstructions that vary widely in severity, ranging from transient functional ileus to meconium ileus with cystic fibrosis, and meconium plug syndrome (MPS). MPS is characterized by a lowlevel intestinal obstruction. The course is typically benign, and the plug is eliminated via rectal stimulation or contrast enema ${ }^{1-4)}$. MPS can be associated with motility disorders including Hirsch sprung's disease and cystic fibrosis ${ }^{5,6)}$. Meconium obstruction of prematurity (MOP) is a clinical entity distinct from meconium ileus and MPS in term neonates ${ }^{1-4)}$. The number of extremely low-birth-weight (LBW) neonates with MOP and intestinal perforation has been increasing because more extremely LBW neonates survive and the live-birth rate of such infants has increased $^{7,8)}$. MOP develops in very LBW infants who develop obstructive symptoms several days after initially passing some meconium $^{3,7,9)}$. MOP is not associated with any underlying disease such as congenital bowel obstruction, necrotizing entero colitis (NEC), or hypothyroidism ${ }^{3,7,9,10}$. MOP in preterm infants is caused by the high viscousness of the meconium and poor bowel motility associated with ganglion immaturity ${ }^{8-11)}$. If the sticky meconium fails to progress, it causes bowel obstruction and feeding cannot be ensued ${ }^{7}$. MOP can be complicated by bowel ischemia or increased mucosal permeability, progressing to perforation, NEC, or sepsis ${ }^{9,10,12,13)}$. MOP is associated with highlevel morbidity or mortality, associated with prolonged hospitalization if not diagnosed and treated appropriately ${ }^{1)}$.

The modalities used to diagnose bowel obstruction, followup, and treat meconium plugs and uncomplicated meconium ileus include plain radiography and contrast enema ${ }^{2,3,14-17)}$; these are based principally on low-quality evidence and expert opinion $^{18)}$. Water-soluble contrast media are hyperosmolar and can loosen a tenacious meconium by drawing large volumes of fluid into the bowel, freeing the meconium and allowing it to pass through the rectum ${ }^{14)}$. This also promotes bowel peristalsis ${ }^{19)}$. Although water-soluble contrast enemas are increasingly given to preterm infants, the overall success rate is only $36 \%$ to $54.5 \%$, and large amounts of contrast media are required ${ }^{15-17,20,21)}$. If medical treatments fail or a bowel perforation develops, surgery is needed. In surgically managed patients, $50 \%$ to $83 \%$ of all perforations were in the distal ileum. Standard medical therapy is less effective in such infants because the enema may not attain the obstructed area ${ }^{1,7)}$.
We speculate that oral nonionic water-soluble contrast media loosen the meconium and promote peristalsis. We administered oral contrast media to seven clinically diagnosed MOP patients whose obstructions were not relieved via conventional mana gement, to avoid risky surgery. We retrospectively evaluated whether oral nonionic water-soluble contrast media relieve MOP.

\section{MATERIALS AND METHODS}

Our Institutional Review Board approved this retrospective study. A total of 385 LBW premature infants were admitted to the neonatal intensive care unit from June 2015 to January 2019. Of these, 67 (17.4\%) were clinically and radiologically diagnosed with $\mathrm{MOP}^{3,7,9,10,15)}$. All the infants had no NEC and weighed less than $1,500 \mathrm{~g}$. Fifty-four patients improved on medical treatment, two underwent surgery due to bowel perforation or unresolved bowel distention. Nine underwent contrast enema (four success ful and five failure to treat MOP). Seven (7/67, 10.4\%; five failed contrast enema and two oral contrast media only) were managed using oral contrast media (Omnipaque350, GE Healthcare, Shanghai, China; $844 \mathrm{mOsm} / \mathrm{kg}$ water).

Premature infants in the neonatal intensive care unit were managed using glycerin and warm saline enemas to prevent the development of meconium-related disease. Abdominal ultrasonography (US) and plain radiography were performed for all MOP patients. Prior to administration of oral contrast media, five patients received sonography-guided water-soluble contrast enemas (cases 1-3, 6, 7) (Iomeron 300, Bracco, BIPSO GmbH, Germany; $521 \mathrm{mOsmol} / \mathrm{kg}$ water) to ensure that the contrast media reaches the ileum. This was not effective in all five patients; therefore, contrast media were administrated orally via a gavage tube. Two patients with gasless abdomens received oral contrast enemas as initial treatment.

Prior to contrast administration, all patients underwent US to rule out NEC and any other bowel abnormality. To ensure that the contrast medium reached the loops of the distal small bowel, the contrast medium was injected manually via a gavage tube in the right anterior oblique position of the infant. The amount of contrast media was equivalent to a feed. Follow-up radiographs were routinely taken 6 and 24 hours after the procedure to evaluate bowel distention and evacuation of the contrast media. We recorded the intervals from birth to initial symptom onset, from birth to the procedure, and from the procedure 
to radiographic improvement, meconium evacuation, and commencement of enteral feeding. We obtained demographic data, maternal histories (presence of pre-eclampsia/eclampsia, gestational diabetes mellitus [GDM], premature rupture of membranes, chorioamnionitis, oligohydramnios, $\mathrm{MgSO}_{4}$, or steroid administration during pregnancy, and mode of delivery), and neonatal clinical data (small for gestational age [SGA], patent ductus arteriosus [PDA], and respiratory distress syndrome [RDS]) from medical records.

\section{RESULTS}

Patient demographics and outcomes of oral contrast media treatment are listed in Table 1. MOP presented as abdominal distention, poor passage of meconium, and feeding intolerance. MOP was diagnosed when a persistent or progressive gaseous bowel distension was noted on plain radiography, together with hypoechoic meconium-filled bowel loops and distended proximal bowel loops on US without NEC $(n=5)$. Two infants had gasless abdomens, in which only the stomach gas was visible, and US showed meconium- filled bowel loops $(\mathrm{n}=2)$. Included patients did not exhibit NEC clinically and radiologically.

We administered the oral contrast media to five male and two female infants. The gestational ages and body weights (median [interquartile range, IQR]) at birth versus at the time of oral contrast media administration were $27^{+5}$ weeks (IQR, $24^{+6}$ to $29^{+2}$ ) and $890 \mathrm{~g}(\mathrm{IQR}, 660$ to 1,055$)$ versus $28^{+1}$ weeks (IQR, $26^{+1}$ to $29^{+5}$ ) and $822 \mathrm{~g}$ (IQR, 610 to 1,040), respectively. The median age of MOP diagnosis was 7 days (IQR, 3.5 to 8.5 ). Two (28.6\%) infants were SGA. RDS (7/7), PDA (6/7), grade 4 germinal matrix hemorrhage
(2/7), and pneumothorax (3/7) were associated with the perinatal period. Pre-eclampsia (4/7) was the most common maternal risk factor; GDM, chorioamnionitis, and oligohydramnios were also associated with MOP. Five of the seven (71.4\%) infants had maternal risk factors and one a history of both maternal GDM and pre-eclampsia.

Two mothers had received $\mathrm{MgSO}_{4}$, and five mothers had received antenatal corticosteroids. Six (85.7\%) infants were born via emergency cesarean section, and one via premature spon taneous vaginal delivery.

Radiography revealed multiple distended intestinal loops without air-fluid interfaces in five cases (cases 1-3, 6, 7) (Figure 1). Two exhibited gasless abdomens, in which only stomach gas was visible (cases 4,5) (Figure 2). Oral contrast medium (median, $2.5 \mathrm{~mL}$ [IQR, 2.25 to 4$]$ ) was administrated at a median age of 8 days (IQR, 6 to 9). MOP was relieved in five patients (71.4\%). Two infants developed vomiting without contrast media progression to the distal bowel loops and were managed surgically. Two infants were diagnosed as having ileal stenosis with microperforation (patient 6) or hypoganglionosis (patient 7). Meconium was evacuated within 1 day in three patients, 2 days in one, and 4 days in one. Feeding commenced within 1 day in two patients, 2 days in two, and 7 days later in one. Radiographic improvements were apparent within 1 day in three patients, 3 days in one, and 5 days in one.

\section{DISCUSSION}

Meconium obstruction in a LBW infant ranges from mild functional ileus to a perforation. The incidence of MOP is unclear but

Table 1. Clinical Characteristics and Outcomes of Oral Contrast Media to Treat Meconium Obstruction in Premature Infants

\begin{tabular}{|c|c|c|c|c|c|c|c|c|c|c|c|}
\hline \multirow{2}{*}{ Case } & \multirow{2}{*}{ Sex } & \multirow{2}{*}{$\begin{array}{l}\text { GA } \\
\text { (wk) }\end{array}$} & \multirow{2}{*}{$\begin{array}{l}\text { BW } \\
(\mathrm{g})\end{array}$} & \multicolumn{2}{|c|}{$\begin{array}{l}\text { On the day of } \\
\text { procedure }\end{array}$} & \multirow{2}{*}{$\begin{array}{c}\text { Sx onset } \\
\text { (d after birth) }\end{array}$} & \multicolumn{4}{|c|}{$\begin{array}{l}\text { Procedure day/time after oral contrast media } \\
\text { (d after birth) }\end{array}$} & \multirow{2}{*}{$\begin{array}{c}\text { Complete } \\
\text { evacuation } \\
\text { of contrast } \\
\text { media }\end{array}$} \\
\hline & & & & GA & BW & & $\begin{array}{c}\text { Contrast } \\
\text { amount (cc) }\end{array}$ & $\begin{array}{l}\text { Meconium } \\
\text { evacuation }\end{array}$ & $\begin{array}{l}\text { Radiological } \\
\text { improvement }\end{array}$ & $\begin{array}{l}\text { Enteral } \\
\text { feeding }\end{array}$ & \\
\hline 1 & $\mathrm{M}$ & $29^{+3}$ & 1,490 & $30^{+5}$ & 1,440 & 3 & $8 / 10$ & 1 & 1 & 1 & 2 \\
\hline 2 & M & $27^{+4}$ & 760 & $28^{+1}$ & 680 & 4 & $5 / 2.5$ & 1 & 1 & 7 & 2 \\
\hline 3 & M & $25^{+6}$ & 890 & $27^{+1}$ & 822 & 9 & $10 / 5$ & 1 & 1 & 1 & 1 \\
\hline 4 & M & $23^{+3}$ & 560 & 25 & 540 & 13 & $13 / 2.5$ & 2 & 5 & 2 & 7 \\
\hline 5 & $\mathrm{~F}$ & $23^{+6}$ & 410 & $24^{+6}$ & 416 & 8 & $8 / 2$ & 4 & 3 & 2 & 7 \\
\hline 6 & M & $29^{+2}$ & 1,060 & $30^{+2}$ & 1,060 & 7 & $7 / 2$ & Surgery & & & \\
\hline 7 & $\mathrm{~F}$ & $29^{+1}$ & 1,050 & $29^{+3}$ & 1,020 & 2 & $2 / 3$ & Surgery & & & \\
\hline
\end{tabular}

Abbreviations: GA, gestational age; BW, birth weight; Sx, symptom. 


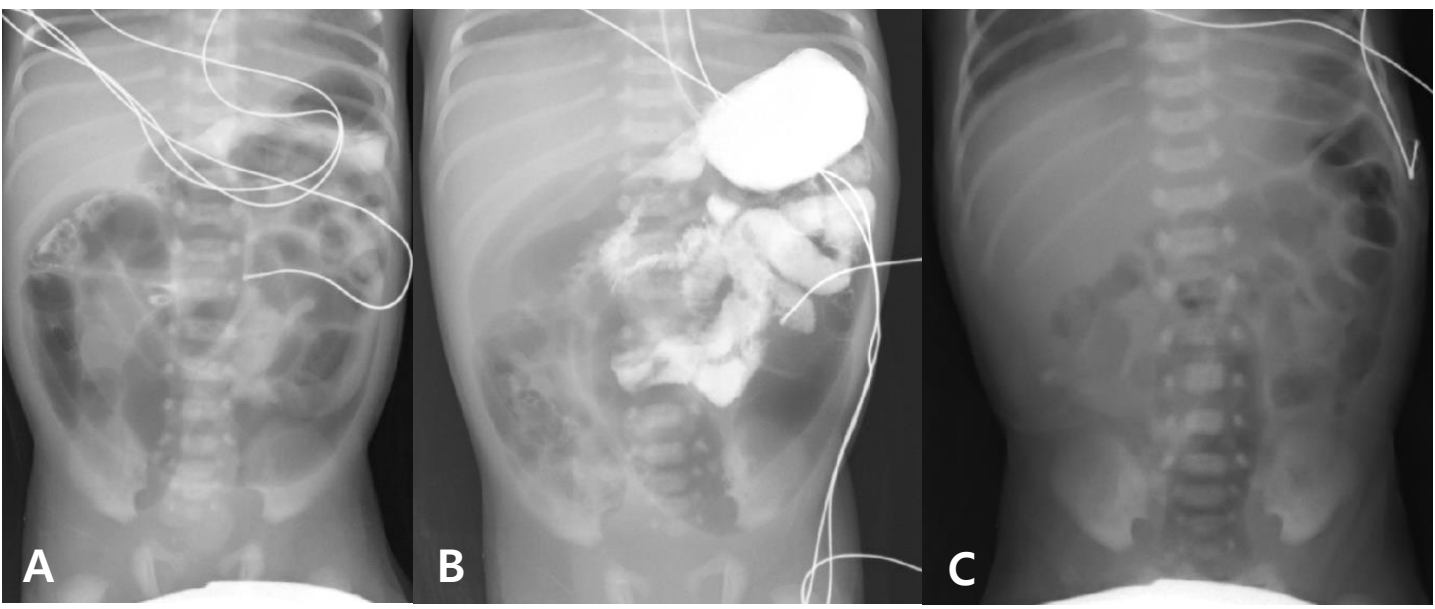

Figure 1. A male infant with a birth weight of $1,490 \mathrm{~g}$ was born at $29^{+3}$ weeks via emergency cesarean section. He exhibited abdominal distention from the 3rd day after birth. He received daily glycerine enemas and a contrast enema on day 7. The abdominal distention persisted. (A) An abdominal radiograph taken after the contrast enema revealed dilated bowel loops with residual contrast in the colon. (B) Oral omnipaque (10 $\mathrm{mL})$ was given via a gavage tube on day $8\left(30^{+5}\right.$ gestational weeks, weight $\left.1,440 \mathrm{~g}\right)$. Within 1 day, he passed the meconium and commenced enteral feeding. (C) Two days after oral contrast medium administration, the bowel gas pattern became normal.

is increasing ${ }^{1,15,21)}$. We found that MOP occurred in $17.4 \%$ of LBW infants.

The differential diagnosis of MOP includes NEC, meconium ileus, Hirschsprung's disease, and intestinal atresia. NEC may clinically resemble MOP. The former is triggered by ischemia due to inspissated meconium syndrome, which is a severe prolonged distention proximal to the meconium obstruction. Meanwhile, the pathophysiology of MOP is unclear. Apart from immaturity of the ganglia, weak peristalsis and excessive water absorption in a hypoperistaltic bowel before birth may render the meconium inspissated ${ }^{1,8-11)}$. Pre- and perinatal risk factors include any condition causing perinatal intestinal hypoperfusion and factors associated with intestinal dysmotility and stasis ${ }^{2,9)}$. Fetal hypoglycemia causes excess glucagon production, in turn decreasing bowel motility ${ }^{1)}$. Maternal hypertension and preeclampsia/eclampsia may trigger prenatal intestinal hypoperfusion $^{2,9)}$. GDM and hypermagnesemia are associated with functional obstruction in premature infants, attributed to depression of intestinal smooth muscle activity and delayed peristalsis ${ }^{1,2,9)}$. Having a lower body weight at birth or at the time of the contrast enema (SGA) reduces the success rate of contrast enema ${ }^{17}$. Five of the seven (71.4\%) infants exhibited maternal risk factors, one of which had a combination of maternal GDM and pre-eclampsia. However, the relationship between risk factors and MOP and the optimal treatment remain to be investigated.

MOP should be clinically suspected when a preterm infant inef- fectively passes a meconium and exhibits progressive abdominal distention and feeding intolerance despite administration of a glycerine enema ${ }^{1,15)}$. Prompt recognition and early aggressive medical treatment are essential to prevent surgical intervention. Perforation develops if the obstruction is not relieved. Delayed diagnosis and an inspissated meconium in the distal ileum are common in infants who develop perforations ${ }^{1)}$.

The use of plain radiography to diagnose large bowel obstruction, followed by hyperosmolar contrast enema for follow-up and treatment, is acceptable. Treatment of meconium-related disease is based principally on consensus opinion of lowquality evidence ${ }^{18)}$. A sonography-guided water-soluble contrast enema remains the treatment of choice to relieve the obstruc tions in meconium-related diseases including MPS, MOP, and meconium ileus ${ }^{1-3,14-17}$. This treatment is both non-invasive and effective. Success depends on the reflux of contrast media into the distal ileum, where an inspissated meconium is located most frequently ${ }^{1,17)}$. Garza-Cox et al. ${ }^{1)}$ reported that $38 \%$ of obstruction sites were in the distal ileum and $10 \%$ in both the distal ileum and colon. Reflux of contrast medium into the distal ileum is a significant predictor of success but does not occur in all cases $^{15-17,20,21)}$, requiring repeat enemas. Although water-soluble contrast enemas are increasingly used to treat preterm infants, the overall success rate is only $36 \%$ to $54.5 \%$, and a large amount of contrast medium is required ${ }^{15-20}$. If medical treatments fail or a bowel perforation develops, surgery is required. Of MOP 
patients, $9 \%$ to $50 \%$ require surgery, and $50 \%$ to $83 \%$ of obstructions occur in the distal ileum ${ }^{1,2,17)}$. Standard medical therapy is less effective in such infants because the enema may not reach the obstructed area ${ }^{1,7)}$. Apart from the ileum, surgically managed patients had obstructions in other small bowel loops and the associated microcolon, which are not resolved via repeated contrast enemas; $38 \%$ to $43 \%$ exhibit an abnormal bowel histopathology ${ }^{1,2,5-7)}$. In this study, two patients (28.6\%) who were surgically managed had abnormal bowel histopathology.

Oral agents are potential adjuncts to enemas. Greenholz et al. ${ }^{2)}$ administered $10 \%$ oral acetylcysteine for 2 weeks to an infant who underwent a gastrografin enema. $\mathrm{N}$-acetylcysteine given via an orogastric tube, as advocated by Noblett ${ }^{14)}$, reduced stool viscosity by $99 \%$ after 6 hours ${ }^{22)}$. Water-soluble contrast media are hyperosmolar; they pull fluid into the intestinal lumen, which hydrates and softens the meconium mass ${ }^{20,23)}$ and promotes bowel peristalsis ${ }^{19)}$. We used an undiluted contrast media with an osmolarity higher than that of normal plasma. We speculate that oral contrast media can assist patients with MOP who do not respond to conventional contrast enemas or in whom the

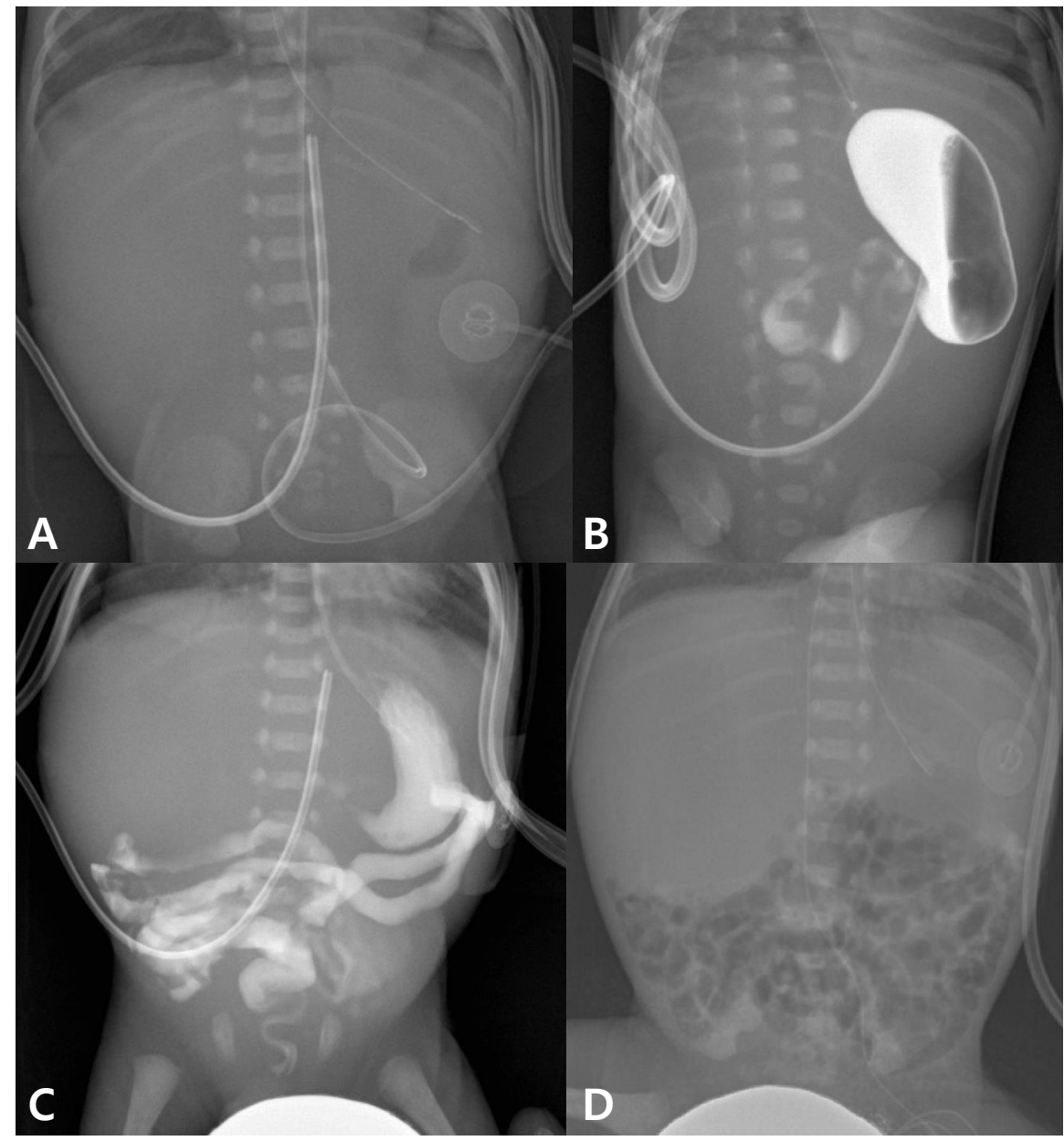

Figure 2. A male infant with a birth weight of $560 \mathrm{~g}$ was born at $23^{+3}$ weeks via emergency cesarean section. He was not small for gestational age and had no maternal risk factors. After passage of some meconium following a glycerine enema (A), no bowel gas was evident (only stomach gas was present). A degree of abdominal distention, poor meconium passage, and a clinical meconium-associated obstruction were suspected. (B) Approximately $2.5 \mathrm{~mL}$ of contrast medium was administrated via a gavage tube on day 13 of life (25 gestational weeks, weight $500 \mathrm{~g}$ ). (C) A 24-hour follow-up radiograph shows contrast medium into the bowel loops (D) contrast medium was completely evacuated by day 7 after administration, and a follow-up radiograph revealed a normal bowel gas pattern. He passed meconium 2 days after the oral contrast media. Enteral feeding commenced on that day. 
meconium is located in small bowel loops. In an effort to avoid the need for surgery, we tested small amounts (median, 2.5 $\mathrm{mL}$, equivalent to a feed) of oral contrast media and found that $71.4 \%$ of patients improved, and $42.9 \%$ of patients evacuated meconium within 1 day and $57.1 \%$ within 2 days. Enteral feeding commenced within 2 days in $57.1 \%$ of responding patients. Oral contrast administration is simple and useful in treating patients with a meconium in the small bowel.

Our study had limitations. It was a single-center, non-controlled retrospective study with a small number of patients. Therefore, a multicenter prospective study with a large number of patients is needed. The contrast media in this study was hyperosmolar. So far, there has been no consensus on the dose and concentration of contrast media in premature infants, requiring further studies.

In conclusion, oral water-soluble contrast media are useful to treat premature infants with meconium obstructions. The procedure has a very low-risk, and we used much less contrast medium than employed for a contrast enema.

\section{ARTICLE INFORMATION}

\section{Ethical statement}

This study was conducted ethically in accordance with the World Medical Association Declaration of Helsinki. The Insti tutional Review Board of Soonchunhyang University Hospital approved this retrospective study (IRB number: SCH 2019-07017-001).

\section{Conflicts of interest}

No potential conflict of interest relevant to this article was reported.

\section{Author contributions}

Conception or design: H.S.H., S.S.K., G.Y.P.

Acquisition, analysis, or interpretation of data: H.S.H., S.S.K., G.Y.P.

Drafting the work or revising: H.S.H.

Final approval of the manuscript: H.S.H.

\section{ORCID}

Hyun Sook Hong https://orcid.org/0000-0003-3210-9740

\section{Acknowledgments}

This work was supported in part by the Soonchunhyang
University Research Fund.

\section{REFERENCES}

1. Garza-Cox S, Keeney SE, Angel CA, Thompson LL, Swischuk LE. Meconium obstruction in the very low birth weight premature infant. Pediatrics 2004;114:285-90.

2. Greenholz SK, Perez C, Wesley JR, Marr CC. Meconium obstruction in markedly premature infant. J Pediatr Surg 1996;31:117-20.

3. Vinograd I, Mogle P, Peleg O, Alpan G, Lernau OZ. Meconium disease in premature infants with very low birth weight. J Pediatr 1983;103:963-6.

4. Amodio J, Berdon W, Abramson S, Stolar C. Microcolon of prematurity: a form of functional obstruction. AJR Am J Roentgenol 1986;146:239-44.

5. Burge D, Drewett M. Meconium plug obstruction. Pediatr Surg Int 2004;20:108-10.

6. Keckler SJ, St Peter SD, Spilde TL, Tsao K, Ostlie DJ, Holcomb GW 3rd, et al. Current significance of meconium plug syndrome. J Pediatr Surg 2008;43:896-8.

7. Kim YJ, Kim EK, Kim ES, Kim HS, Choi JH, Cheon JE, et al. Recognition, diagnosis and treatment of meconium obstruction in extremely low birth weight infants. Neonatology 2012;101:172-8.

8. Kubota A, Shiraishi J, Kawahara H, Okuyama H, Yoneda A, Nakai $\mathrm{H}$, et al. Meconium-related ileus in extremely low-birthweight neonates: etiological considerations from histology and radiology. Pediatr Int 2011;53:887-91.

9. Dimmitt RA, Moss RL. Meconium diseases in infants with very low birth weight. Semin Pediatr Surg 2000;9:79-83.

10. Krasna IH, Rosenfeld D, Salerno P. Is it necrotizing enterocolitis, microcolon of prematurity, or delayed meconium plug? A dilemma in the tiny premature infant. J Pediatr Surg 1996;31:855-8.

11. Yoo SY, Jung SH, Eom M, Kim IH, Han A. Delayed maturation of interstitial cells of Cajal in meconium obstruction. J Pediatr Surg 2002;37:1758-61.

12. Chan KL, Ng SP, Chan KW, Wo YH, Tam PK. Pathogenesis of neonatal necrotizing enterocolitis: a study of the role of intraluminal pressure, age and bacterial concentration. Pediatr Surg Int 2003;19:573-7.

13. Ein SH, Shandling B, Reilly BJ, Stephens CA. Bowel perforation with nonoperative treatment of meconium ileus. J Pediatr Surg 1987;22:146-7.

14. Noblett HR. Treatment of uncomplicated meconium ileus by Gastrografin enema: a preliminary report. J Pediatr Surg 1969;4: 190-7.

15. Emil S, Nguyen T, Sills J, Padilla G. Meconium obstruction in extremely low-birth-weight neonates: guidelines for diagnosis and management. J Pediatr Surg 2004;39:731-7. 
16. Goo HW, Kim KS, Kim EA, Pi SY, Yoon CH. Sonography-guided gastrografin enema for meconium plug syndrome in premature newborns: preliminary results. J Korean Radiol Soc 2004;50:2818.

17. Cho HH, Cheon JE, Choi YH, Lee SM, Kim WS, Kim IO, et al. Ultrasound-guided contrast enema for meconium obstruction in very low birth weight infants: factors that affect treatment success. Eur J Radiol 2015;84:2024-31.

18. Carroll AG, Kavanagh RG, Ni Leidhin C, Cullinan NM, Lavelle LP, Malone DE. Comparative effectiveness of imaging modalities for the diagnosis of intestinal obstruction in neonates and infants: a critically appraised topic. Acad Radiol 2016;23:559-68.

19. Haiden N, Norooz F, Klebermass-Schrehof K, Horak AS, Jilma B, Berger A, et al. The effect of an osmotic contrast agent on com- plete meconium evacuation in preterm infants. Pediatrics 2012; 130:e1600-6.

20. Copeland DR, St Peter SD, Sharp SW, Islam S, Cuenca A, Tolleson JS, et al. Diminishing role of contrast enema in simple meconium ileus. J Pediatr Surg 2009;44:2130-2.

21. Karimi A, Gorter RR, Sleeboom C, Kneepkens CM, Heij HA. Issues in the management of simple and complex meconium ileus. Pediatr Surg Int 2011;27:963-8.

22. Burke MS, Ragi JM, Karamanoukian HL, Kotter M, Brisseau GF, Borowitz DS, et al. New strategies in nonoperative management of meconium ileus. J Pediatr Surg 2002;37:760-4.

23. Carlyle BE, Borowitz DS, Glick PL. A review of pathophysiology and management of fetuses and neonates with meconium ileus for the pediatric surgeon. J Pediatr Surg 2012;47:772-81. 